\title{
PROGRAMMABLE CURRENT-CONVEYOR-BASED OSCILLATOR EMPLOYING GROUNDED RESISTORS AND CAPACITORS
}

\author{
MUHAMMAD TAHER ABUELMA'ATTI, ABDULRAHMAN \\ KHALAF AL-ALI AND MOHAMED AHSAN \\ King Fahd University of Petroleum and Minerals, Box 203, Dhahran 31261, Saudi Arabia \\ (Received January 18, 1995; in final form April 3, 1995)
}

\begin{abstract}
A new programmable oscillator using two negative first-generation current-conveyors, three grounded capacitors, and two grounded resistors is presented. The proposed circuit enjoys independent oscillation control through a single grounded resistor and independent frequency control through a single grounded capacitor. Replacing one of the grounded resistors by a digitally programmable resistor results in a programmable oscillator.
\end{abstract}

\section{INTRODUCTION}

In a recent letter, Chang [1] proposed a single-resistance-controlled, currentconveyor-based oscillator. The proposed circuit uses three grounded resistors and two grounded capacitors and enjoys independent oscillation and frequency control. However, the major disadvantage of the circuit is the use of three current conveyors, two of them are positive second-generation current-conveyors (CCII+) and the third is a negative first-generation current-conveyor (CCI-).

The purpose of this paper is to propose a new current-conveyor-based singleelement controlled oscillator using only two CCI-, two grounded resistors, and three grounded capacitors. The circuit enjoys independent control of oscillation and frequency and can be easily converted to a voltage-controlled oscillator. By replacing one of the grounded resistors by an operational transconductance amplifier (OTA)-based resistor a digitally programmable oscillator can be realized.

\section{PROPOSED CIRCUIT}

The proposed circuit is shown in Fig. 1. Assuming ideal CCI- with $v_{x}=v_{y}, i_{x}=$ $i_{y}=i_{z}$ routine analysis shows that the characteristic equation of the circuit can be expressed as

$$
\mathrm{s}^{2} \mathrm{C}_{1} \mathrm{C}_{2}+\mathrm{sG}_{4}\left(\mathrm{C}_{3}-\mathrm{C}_{2}\right)+\mathrm{G}_{3} \mathrm{G}_{4}=0
$$




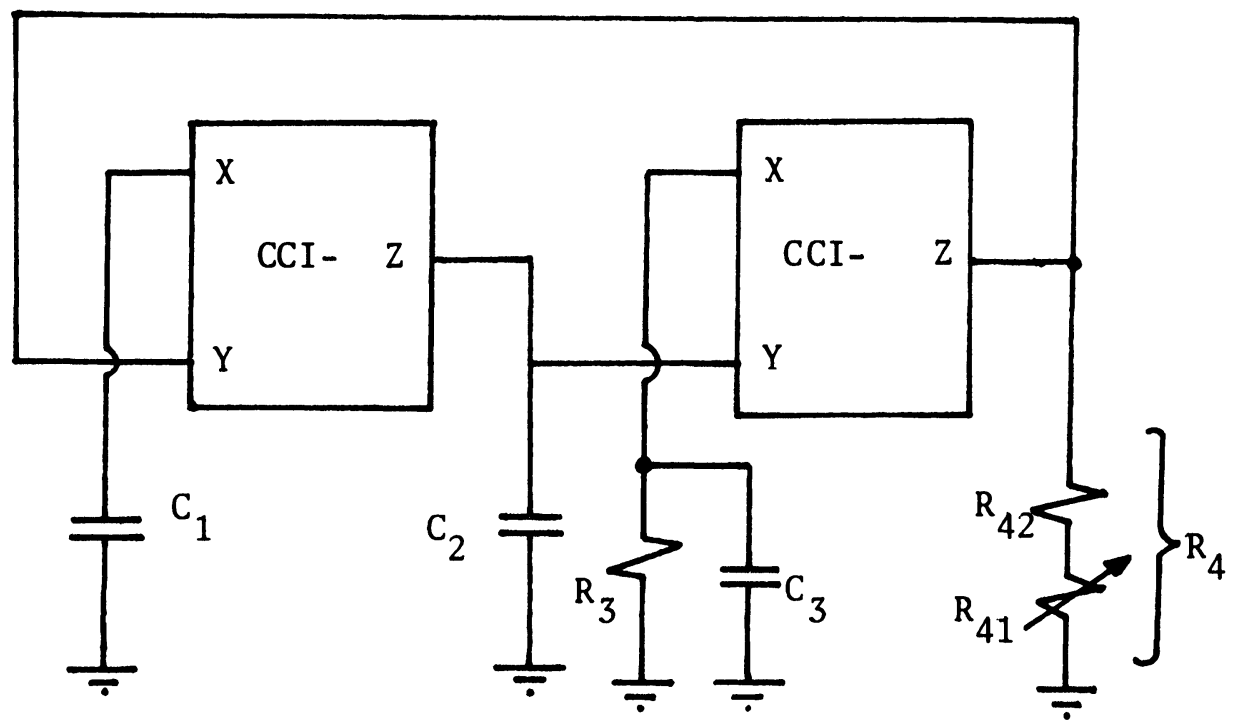

FIGURE 1 Proposed current-conveyor sinusoidal oscillator circuit.

Using (1), the condition of oscillation and the frequency of oscillation can be expressed as

$\omega_{0}^{2}=\frac{1}{C_{1} C_{2} R_{3} R_{4}}$

and

$\mathrm{C}_{2}=\mathrm{C}_{3}$

From (2) and (3), it is easy to see that the frequency of oscillation can be adjusted by tuning $R_{3}$ or $R_{4}$ or $C_{1}$ without disturbing the condition of oscillation while the condition of oscillation can be adjusted by tuning $C_{3}$ without disturbing the frequency of oscillation. Thus, the circuit enjoys independent oscillation and frequency control.

By defining the passive sensitivity of a parameter $\mathrm{F}$ to the element of variation $x_{\mathrm{i}}$ by

$\mathrm{S}_{x_{i}}^{\mathrm{F}}=\frac{\mathrm{dF}}{\mathrm{dx}_{\mathrm{i}}} \frac{\mathrm{x}_{\mathrm{i}}}{\mathrm{F}}$

it is easy to show that the sensitivity of the frequency of oscillation $\omega_{o}$ to the variation in passive elements is given by

$\mathrm{S}_{\mathrm{C}_{1}}^{\omega_{0}}=\mathrm{S}_{\mathrm{C}_{2}}^{\omega_{0}}=\mathrm{S}_{\mathrm{R}_{3}}^{\omega_{0}}=\mathrm{S}_{\mathrm{R}_{4}}^{\omega_{0}}=-\frac{1}{2}$ 


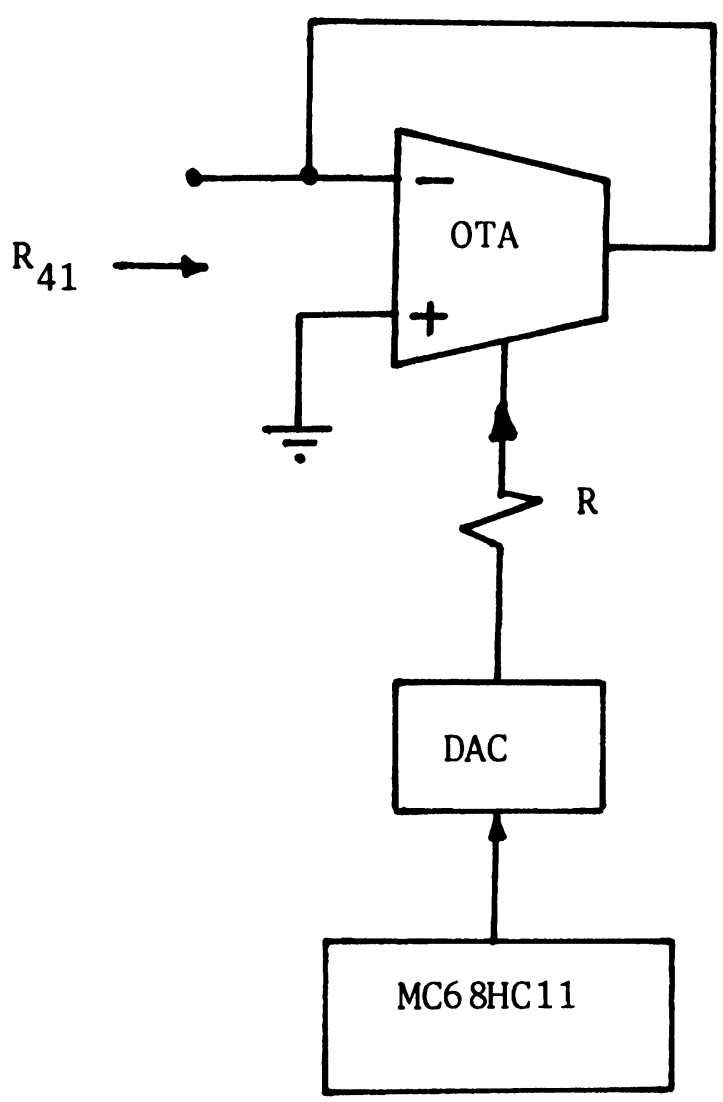

FIGURE 2 Operational-transconductance-amplifier-based realisation of a programmable grounded resistor.

Thus, the $\omega_{0}$-sensitivity to passive elements is low.

The resistor $R_{41}$ can be realized using the operational transconductance amplifier (OTA)-based resistor shown in Fig. 2. Assuming ideal OTA with $i_{o}=g_{m} \nu_{\text {inpuv }}$ where $i_{o}$ is the output current, $g_{m}=I_{A B C} / 2 V_{T}$ is the transconductance of the OTA, $I_{A B C}$ is the auxillary bias current of the OTA, $V_{T}$ is the thermal voltage, and $\nu_{\text {input }}$ is the input voltage of the OTA. By obtaining the current $I_{A B C}$ from the output of the microcontroller, one can see that the frequency of oscillation can be digitallyprogrammed.

\section{PROPOSED SYSTEM HARDWARE}

The hardware architecture consists of a microcontroller, an 8-bit digital-to-analog converter (DAC), an OTA, and two CCI-. The microcontroller is a single chip 8-bit microprocessor that monitors and controls the frequency of oscillation. It has a built-in 256-byte RAM, an 8-kilobyte EPROM, 512-byte EEPROM, 29 inputoutput digital ports, and an 8-channel 8-bit ADC. Through the microcontroller, the 
user is provided complete control over the frequency of oscillation of the oscillator. The user would make his selection through a software menu. The microcontroller would then change the bias current of the OTA, thus changing the value of the resistance $R_{4}$ and thereby changing the frequency of oscillation. The 8-bit DAC provides 256 digital combinations and allows fine tuning and, hence, more programming options. The heart of the system is the MC68HC11 microprocessor. It is used in the single chip mode which requires no external storage. The RAM is used for temporary storage of data processing. The EPROM is used to host the main program that controls the whole procedure. Having the EPROM makes it easy to change the software algorithms for future modification and reboot the new program without adding any new components to the system.

The software for the system was developed in Motorola assembly language. The flowchart shown in Fig. 3 outlines the steps involved. Upon reset, the microprocessor reads the user option and then commands the right digital word to the ADC, which changes the biasing voltage and, hence, the biasing current $I_{A B C}$ of the OTA. This changes the value of the resistance $R_{4}$ and, hence, the frequency of oscillation

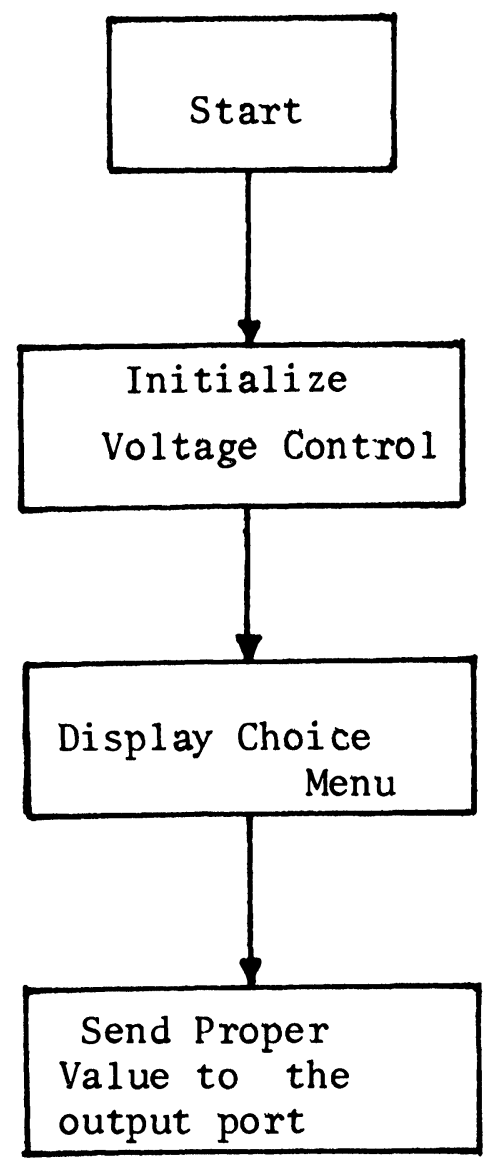

FIGURE 3 Software flow-chart. 


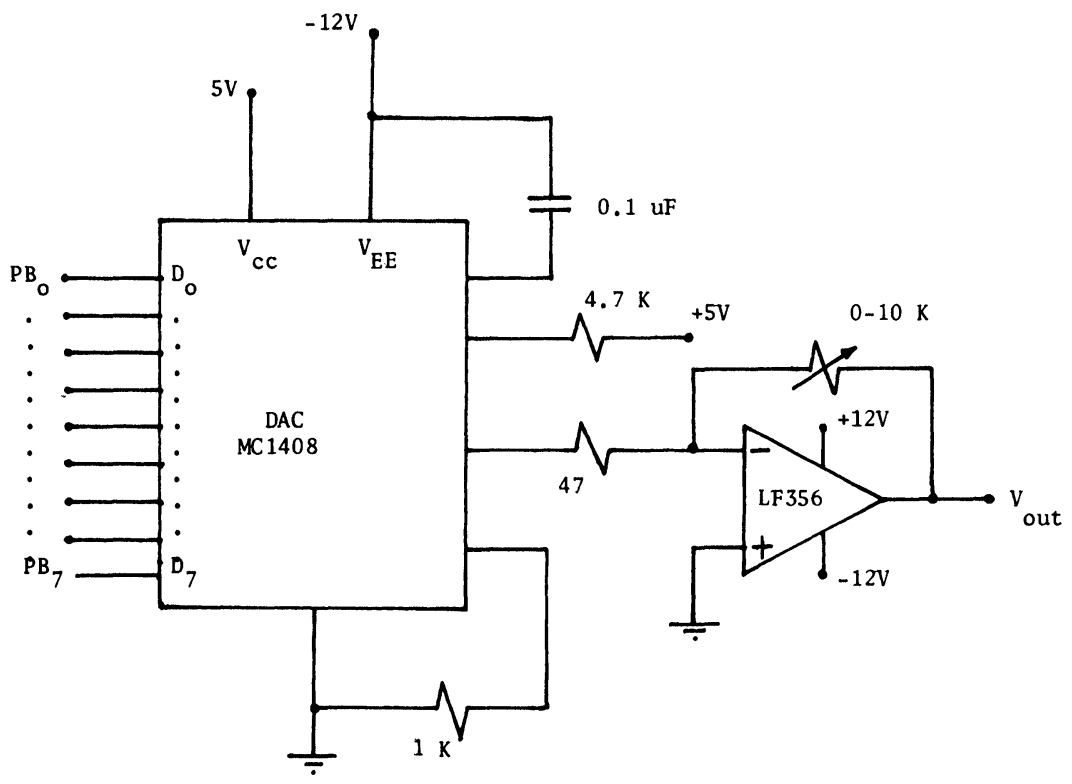

FIGURE 4 Digital-to-analog converter circuitry.

of the oscillator. The menu displayed for the user allows very fine change of the voltage. The voltage levels are between 0.0 to $9.1 \mathrm{~V}$ and they can be changed in 256 steps.

\section{EXPERIMENTAL RESULTS}

The proposed circuit is designed to give an output frequency in the range of 8-10 $\mathrm{MHz}$. This range can be controlled in 256 steps with $1 \mathrm{LSB}$ corresponding to $\approx$ $7 \mathrm{kHz}$. Higher resolutions, and consequently, lower errors can be obtained by using the 10-, 12- or even 16-bit DAC. For example, using a 16-bit DAC leads to a frequency step of $\approx 29 \mathrm{~Hz}$. The DAC circuit connections are shown in Fig. 4 . The CCI- was implemented using the circuit shown in Fig. 5 [2] built around the OTA CA3080 and the operational amplifier LF356. Although there are other higher performance CCI- realizations, the kernel of the work presented in this paper does not depend on the particular implementation of the CCI-. The CA3080 was also used for realizing the digitally programmable resistance $R_{41}$.

A typical output waveform is shown in Fig. 6. The circuit is designed for an output frequency in the range $7.710 \mathrm{MHz}$ to $9.826 \mathrm{MHz}$ when the DAC output is changing from 00 to $\mathrm{FF}$.

\section{CONCLUSION}

In this paper a new current-conveyor-based oscillator has been presented. The circuit uses two negative first-generation current conveyors, two grounded resistors, 


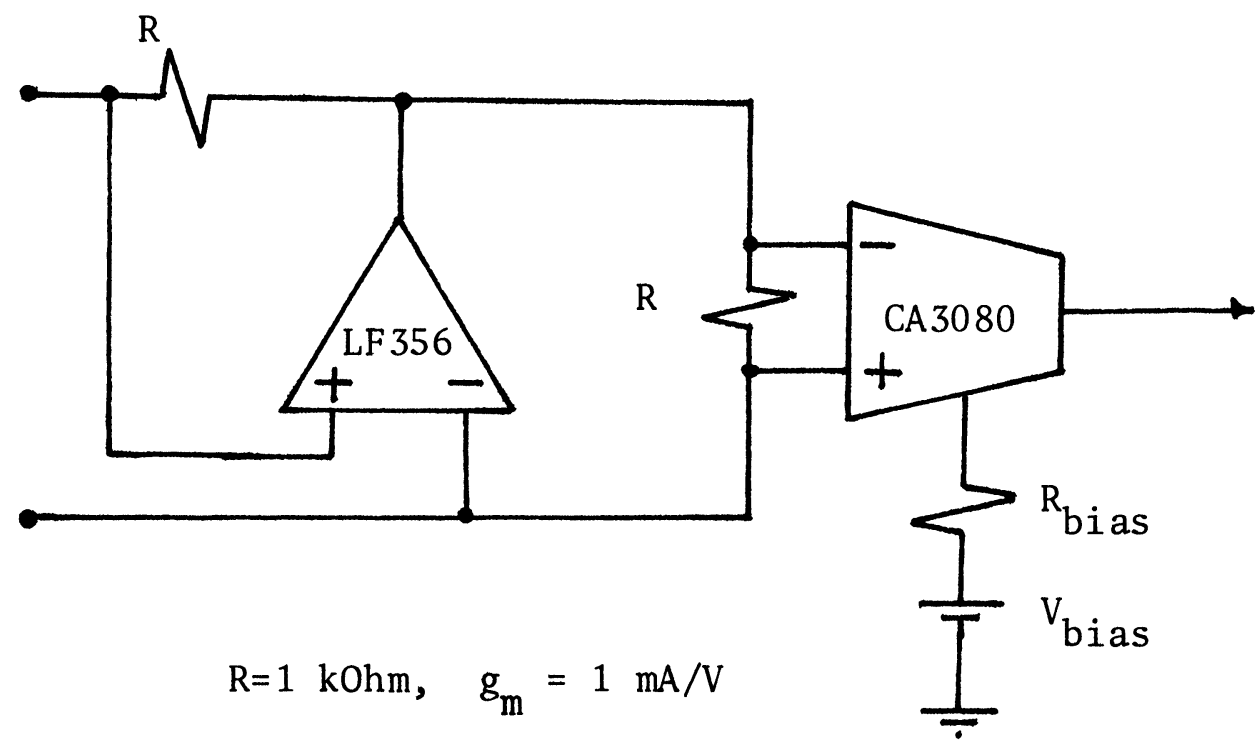

FIGURE 5 Realization of the CCI-[2].

and three grounded capacitors. The circuit enjoys low sensitivities to passive components and independent control of the frequency and the condition of oscillation. The use of grounded capacitors makes the circuit attractive for integration and the use of grounded resistors for independent control of the frequency of oscillation makes the circuit attractive for the realization of voltage controlled oscillators. The circuit can be easily interfaced to a microcontroller,

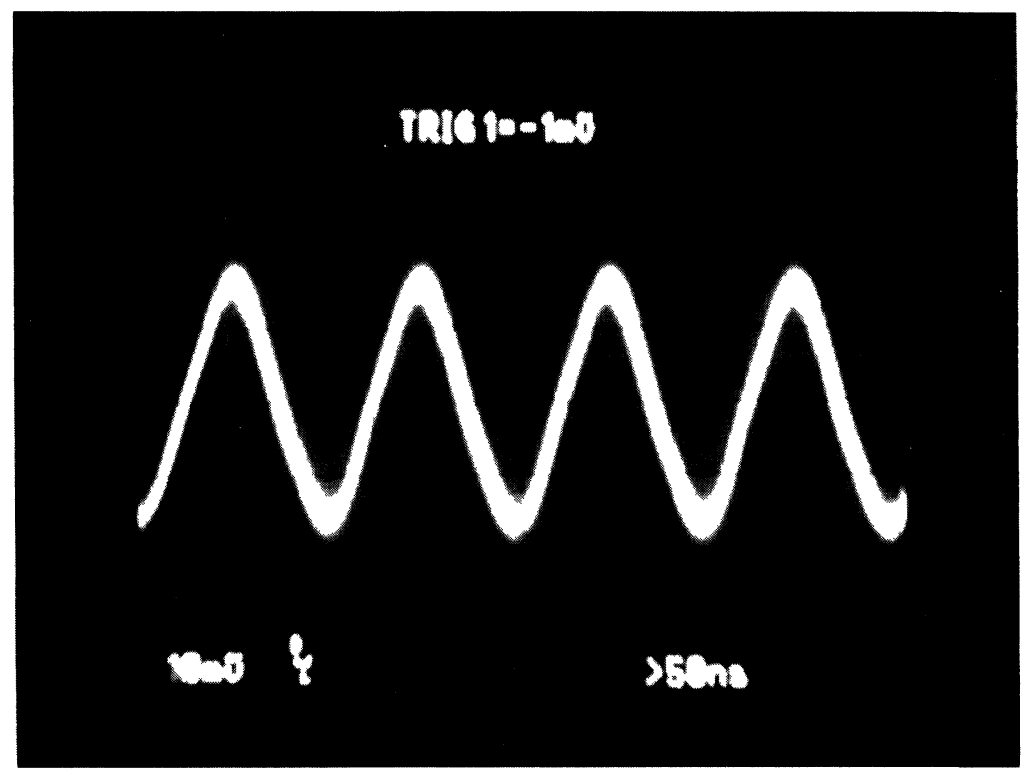

FIGURE 6 A typical output waveform obtained from the circuit of Fig. 1. 
which can monitor and control the frequency of oscillation. Another attractive feature of the circuit is the use of two similar current conveyors. Thus, the circuit appears to be very attractive for integration.

\section{REFERENCES}

1. C.-M. Chang, Novel current-conveyor-based single-resistance-controlled/voltage-controlled oscillator employing grounded resistors and capacitors, Electronics Letters, Vol. 30, 1994, pp. 181-183.

2. R. Senani, Novel circuit implementation of current conveyors using O.A. and an O.T.A., Electronics Letters, Vol. 16, 1980, pp. 2-3. 

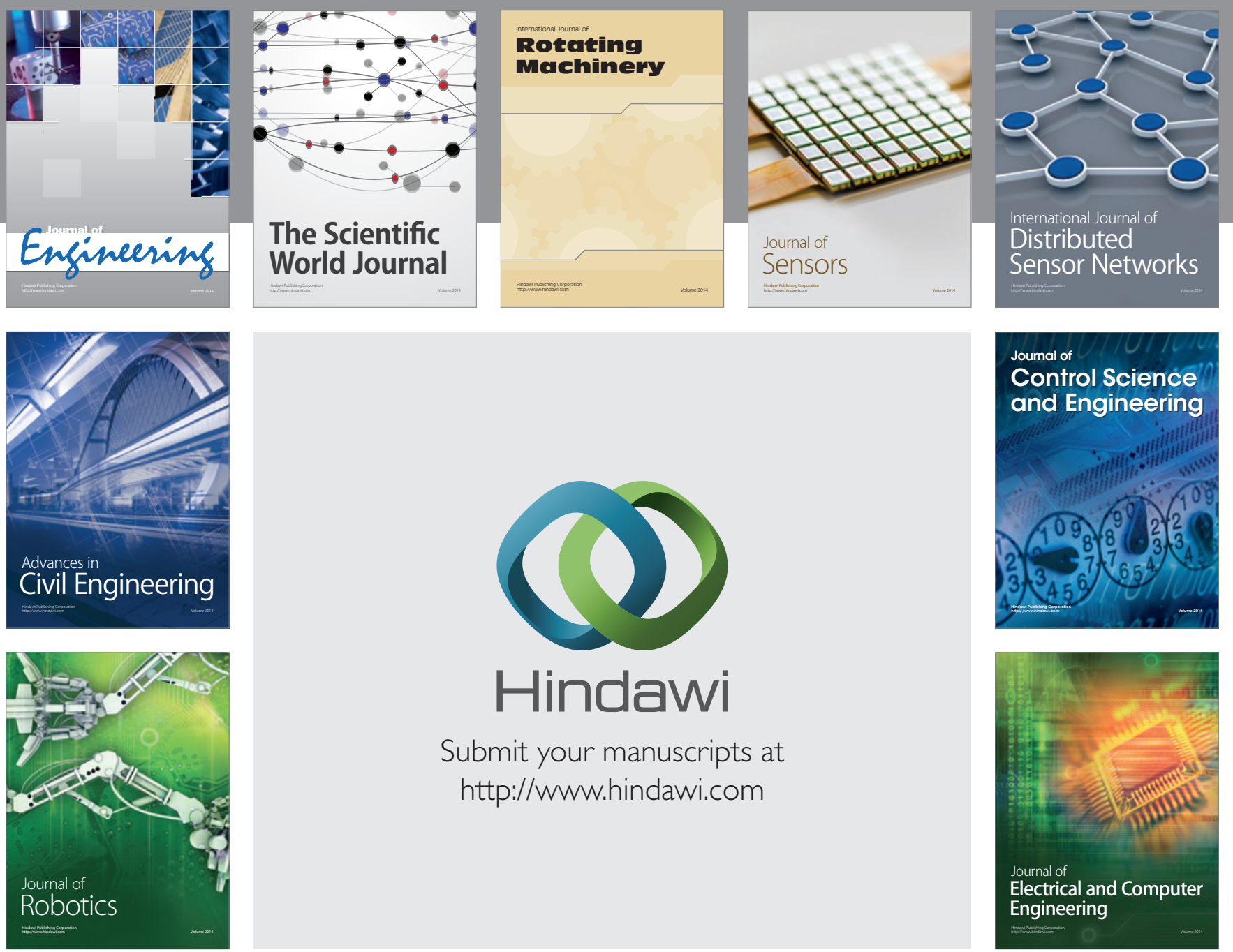

Submit your manuscripts at

http://www.hindawi.com
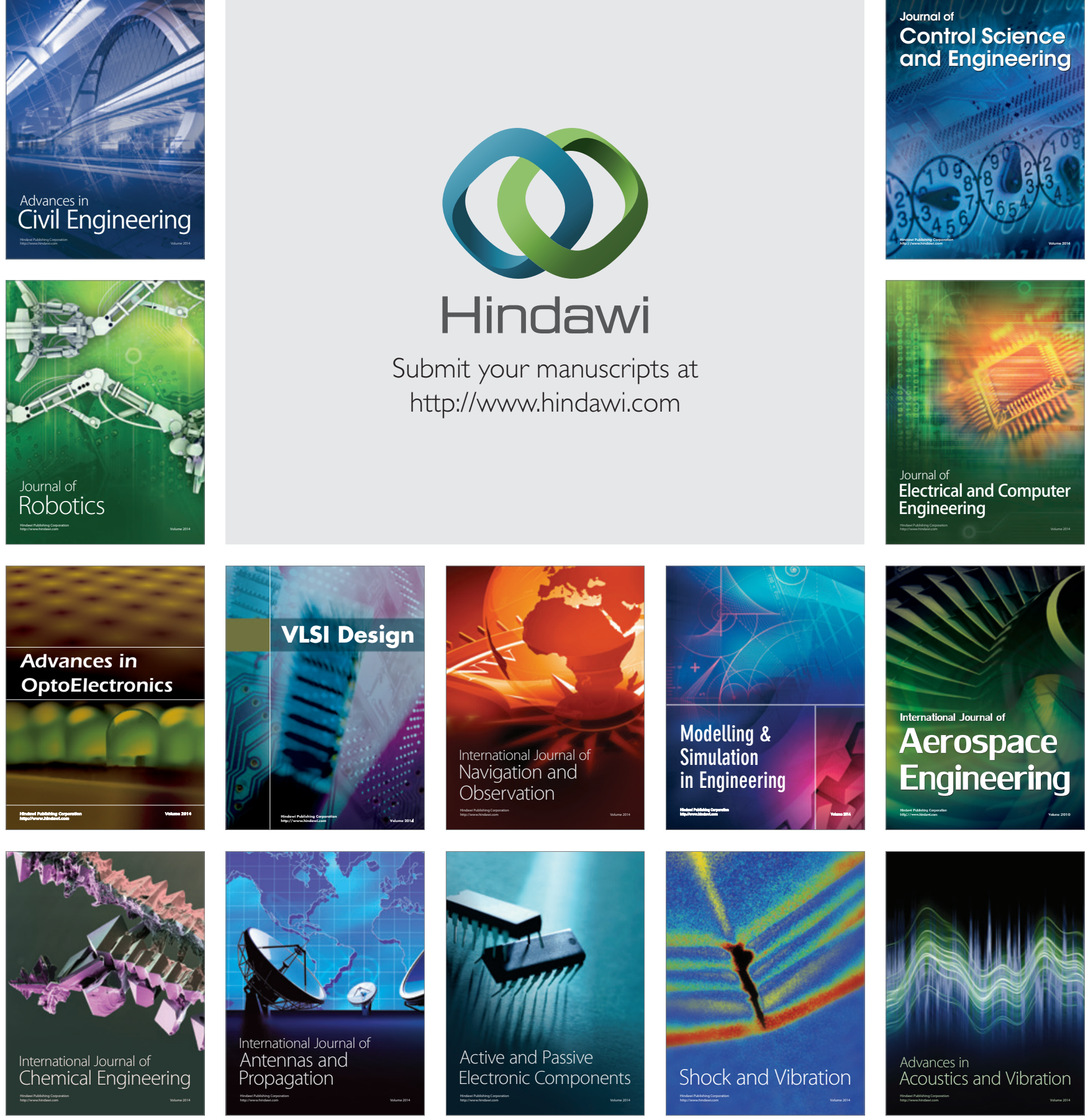